Supporting Information

\title{
Selective interactions between free-atom-like $d$-states in single-atom alloy catalysts and near-frontier molecular orbitals
}

\author{
Taylor D. Spivey ${ }^{1,2}$ and Adam Holewinski*1,2
}

${ }^{1}$ Department of Chemical and Biological Engineering, University of Colorado, Boulder, CO 80303

${ }^{2}$ Renewable and Sustainable Energy Institute, University of Colorado, Boulder, CO 80303

\section{Contents}

Periodic trends in $d$-band characteristics and selected pDOS plots for SAAs $\ldots \ldots \ldots \ldots \ldots \ldots \ldots . \ldots 2$

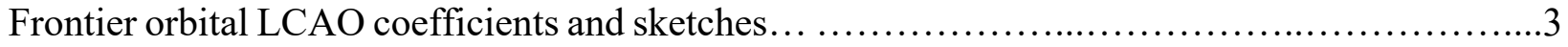

Interaction energies for each MO with $d$-band in each binding mode........................4

Impact of $d$-band functional form on selectivity map....................................

Selectivity model outputs with period and filling dependent coupling elements ................5

Selectivity model visualization with DFT-computed energy differences .....................6

Adsorption geometries and orbital pDOS: ald-mode................................. 7

Adsorption geometries and orbital pDOS: ene-mode $\ldots \ldots \ldots \ldots \ldots \ldots \ldots \ldots \ldots \ldots \ldots \ldots \ldots \ldots \ldots$

Visualization of individual orbital shifts near resonance and model outputs vs. MO separation...9

Molecular orbitals of various $\alpha, \beta$-unsaturated aldehydes................................ 9

Molecular orbitals of down-pathway intermediates for crotonaldehyde reduction.................10

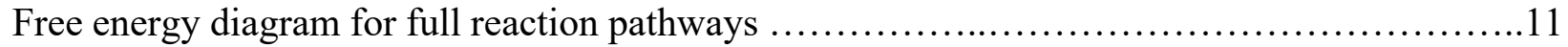

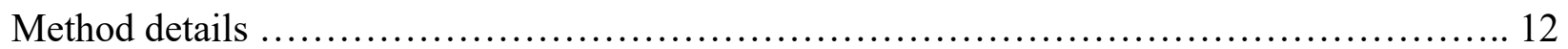

Coupling element angular dependence plots......................................... 15

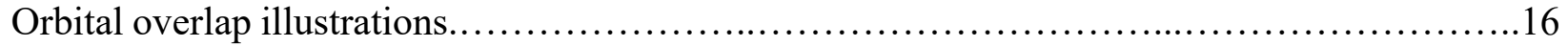

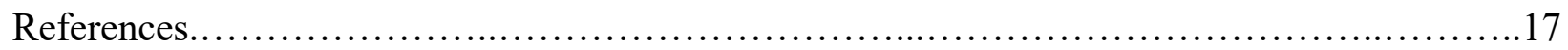


A

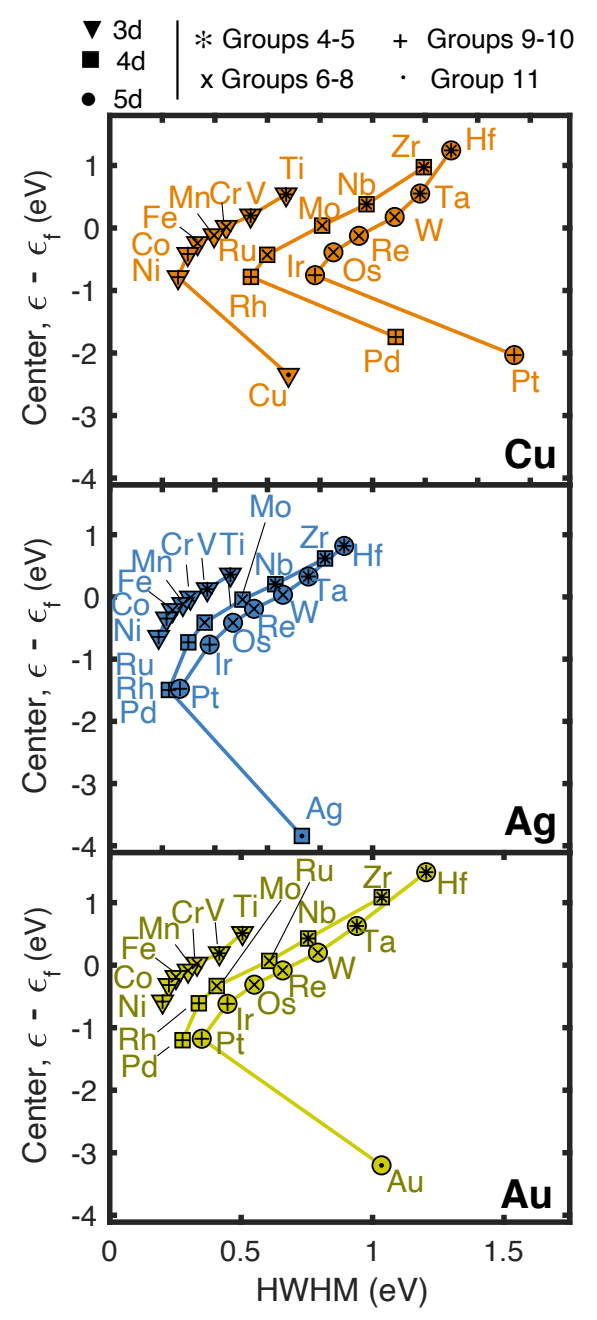

B

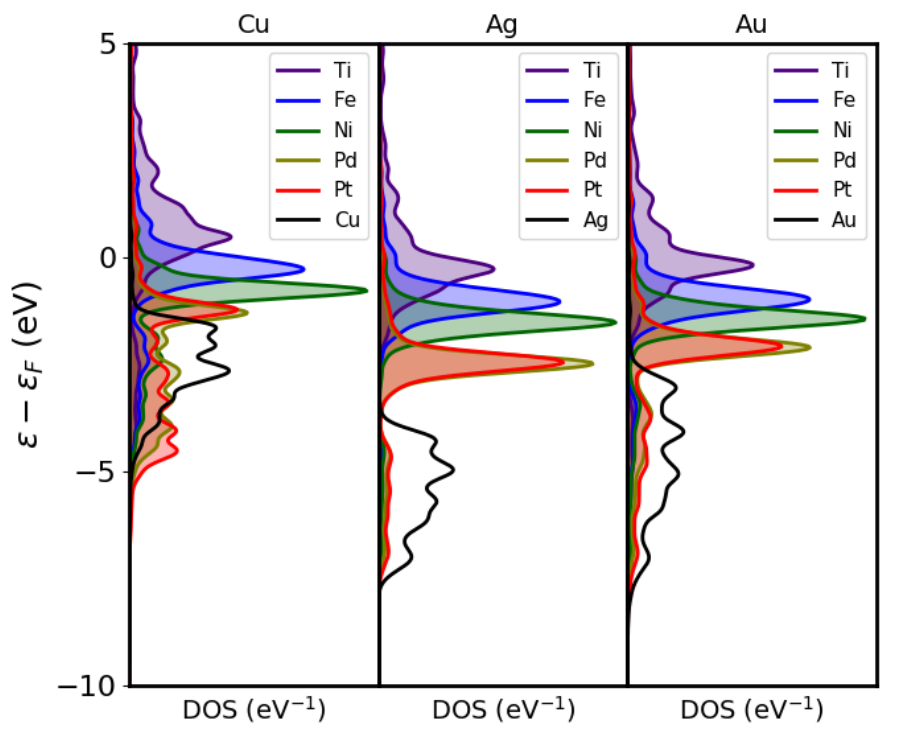

Figure S1: (A) $d$-band center and width (Lorentzian fit HWHM) of all SAAs formed between $\mathrm{Cu}$, $\mathrm{Ag}$, and Au hosts and group 4-10 transition metals. Center and width both decrease when moving across groups within a period (left to right in the periodic table, marker labels: $*_{,}, x_{,}+\cdot$ ), while width increases moving down periods within a group (marker shapes: $\mathbf{\nabla}, \mathbf{\square}, \bullet$ ). Late $4 d$ and $5 d$ elements within $\mathrm{Cu}$ hosts show anomalous hybridization with the host as can be seen with $\mathrm{Pd} / \mathrm{Cu}$ and $\mathrm{Pt} / \mathrm{Cu}$ breaking the trend in width. (B) $d$-projected DOS for surface atoms in a variety of singleatom-alloys. Guest elements are selected to illustrate the trends across a period and down a group of the periodic table: Ti (purple), Fe (blue), Ni (green), Pd (dark yellow) and Pt (red). Host metals are $\mathrm{Cu}, \mathrm{Ag}$, and $\mathrm{Au}$, as labeled. Each host is shown with unshaded black curves. 


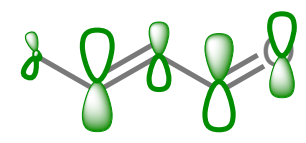

LUMO

$\mathrm{C}-\mathrm{C}$ and $\mathrm{C}-\mathrm{O} \pi^{*}$ system<smiles>CC(=O)OCC=O</smiles>

HOMO-1

C-C bonding $\pi$<smiles>O=CC=CC(=O)O</smiles>

HOMO-3

$C-O$ bonding $\pi$

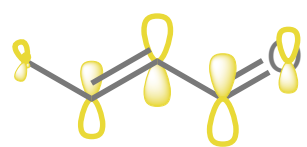

LUMO+1

$\pi^{*}$ system

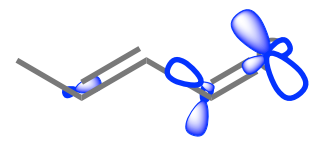

HOMO

$p$ - nonbonding

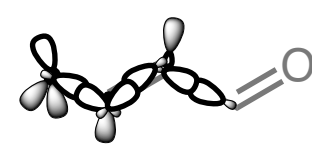

HOMO-2

$\sigma$ system

\begin{tabular}{|c|c|c|c|c|c|c|}
\hline & HOMO-3 & HOMO-2 & HOMO-1 & HOMO & LUMO & LUMO \\
\hline H4 1s & 0.001 & 0.000 & 0.000 & 0.002 & 0.000 & \\
\hline $\mathrm{H} 4 \mathrm{is}$ & 0.058 & 0.000 & 0.038 & 0.000 & 0.036 & \\
\hline $\mathrm{H} 4 \mathrm{1s}$ & 0.000 & 0.022 & 0.032 & 0.000 & 0.031 & \\
\hline $2 s$ & 0.003 & 0.000 & 0.000 & 0.001 & 0.000 & \\
\hline & 0.003 & 0.000 & 0.000 & 0.003 & 0.000 & \\
\hline $2 p z$ & 0.069 & 0.000 & 0.033 & 0.000 & 0.000 & \\
\hline $2 p x$ & 000 & 0.012 & 0.000 & 0.001 & 0.000 & \\
\hline $\begin{array}{ll}\mathrm{H} 3 & 1 \mathrm{~s} \\
\end{array}$ & & 0.000 & .000 & 0.001 & 0.000 & \\
\hline $2 s$ & 01 & 0.000 & 0.000 & 0.000 & 0.000 & \\
\hline $2 p y$ & 0.032 & 0.000 & 0.000 & 0.000 & 0.000 & \\
\hline $2 p z$ & 000 & 0.074 & $\underline{0.294}$ & 0.000 & 0.425 & \\
\hline $2 p x$ & & 0.001 & 001 & 0.008 & 0.002 & \\
\hline $\begin{array}{ll}H 2 & 1 s \\
\end{array}$ & 003 & 0.000 & 0.000 & 0.004 & 0.000 & \\
\hline $2 s$ & 053 & 0.000 & 0.000 & 0.048 & 0.000 & \\
\hline $0^{2 p y}$ & 0.000 & $\underline{0.312}$ & 0.000 & 0.073 & 0.000 & \\
\hline $2 p z$ & & 0.000 & $\underline{0.286}$ & 0.000 & 0.103 & $U$ \\
\hline $2 p x$ & & 0.000 & & 0.001 & 0.001 & 0.00 \\
\hline $\begin{array}{ll}H 1 & 1 s\end{array}$ & & 0.0 & 000 & 0.128 & 0.000 & 0.06 \\
\hline $2 s$ & & 000 & 000 & 0.000 & 0.000 & 0.00 \\
\hline C1 $2 p y$ & 0.017 & 0.000 & 0.000 & 0.000 & 0.000 & 0.00 \\
\hline $2 p z$ & & & & 0.000 & $\underline{0.325}$ & $\pi$ \\
\hline $2 p x$ & & 0.0 & & 0.001 & 0.000 & $\pi$ \\
\hline $2 s$ & & $\underline{0.296}$ & 000 & 0.000 & 0.000 & \\
\hline $2 p y$ & & & 0.000 & $\underline{0.635}$ & 0.000 & \\
\hline & & 0.010 & 214 & 0.001 & $\underline{0.388}$ & \\
\hline & & & & & & \\
\hline
\end{tabular}

Figure S2: Schematic illustration of LCAO orbitals based on atomic orbital coefficients. Squared coefficients are displayed in the table, with significant contributions (values $>0.05$ ) bolded. 

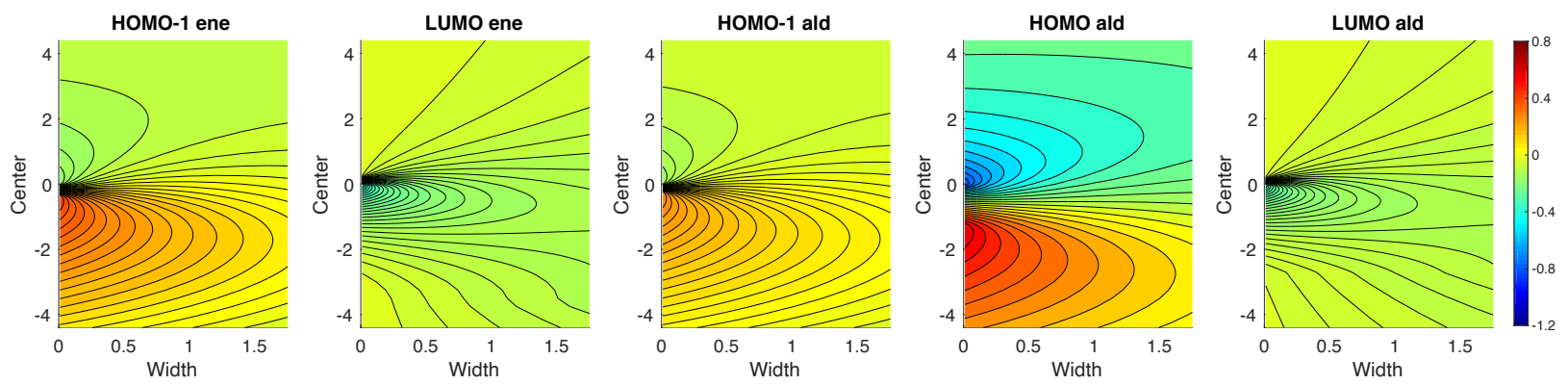

Figure S3: Change in one-electron energy (see methods) upon adsorption for each relevant MO of crotonaldehyde with $d$-band center and width (HWHM) in each binding mode. The LUMO contribution is small and similar in both modes. Color scale in $\mathrm{eV}$.

A

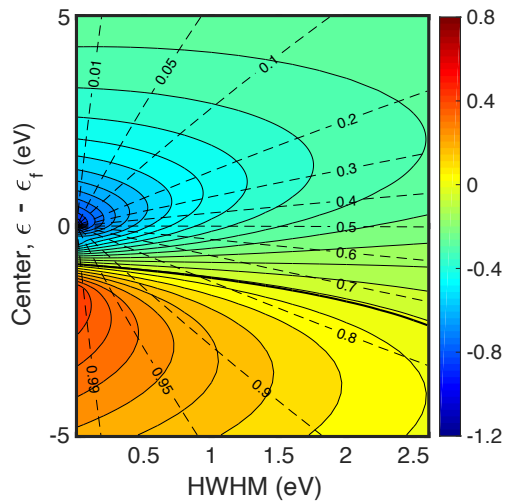

B

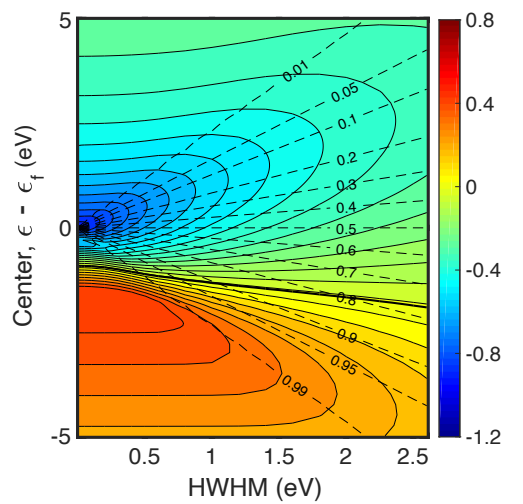

C

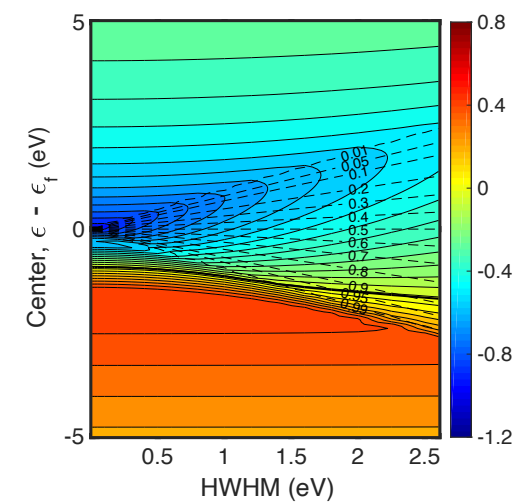

Figure S4: Difference in interaction energy with each adsorbate level $\left(\Delta E_{D}^{\text {ald }}-\Delta E_{U}^{\text {ene }}\right)$ vs. $d$-band characteristics assuming (A) Lorentzian, (B) Gaussian, and (C) semi-elliptical $d$-band $\left(r_{d}=1 \AA\right)$ with LCAO and tight binding parameters for crotonaldehyde. Narrowed SAA states were best fit with a Lorentzian, while monometallics are better described by Gaussian or elliptical functions. Dotted lines represent constant band filling $(f)$. Color scale in $\mathrm{eV}$. 


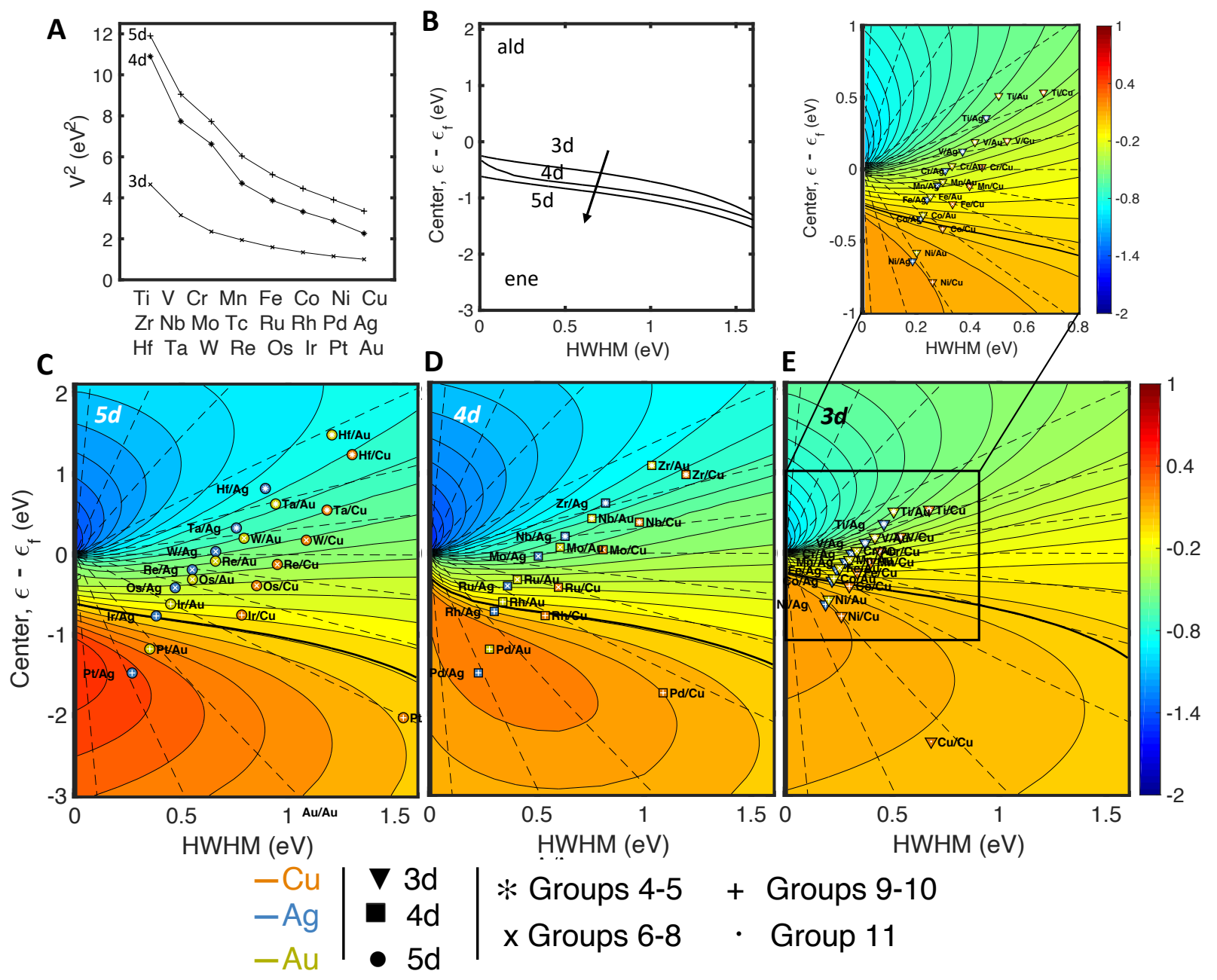

Figure S5: (A) Coupling element ratio (relative to $\mathrm{Cu}$ ) for transition metals (data from Ref. 19) These values were interpolated (based on band-filling, see methods below) to assign periodspecific coupling elements and thus generate selectivity maps that are more characteristic of specific elemental compositions. (B) Overlay of boundary contours for $\Delta \Delta \mathrm{E}=0$, showing trend with period toward lower $d$-center at a given $\Delta \Delta \mathrm{E}$. (C)-(E): Selectivity maps for $5 d, 4 d$, and $3 d$ SAAs, respectively. These are analogous to Figure $4 \mathrm{~A}$ of the main text but account for periodic trends in the spatial extent of $d$-orbitals. Inset: Enlarged region containing most $3 d$ SAAs. Legend applies to all three maps. Color scale in $\mathrm{eV}$. A parity plot for all data points above is given in Figure $4 \mathrm{~B}$ of the main text. 

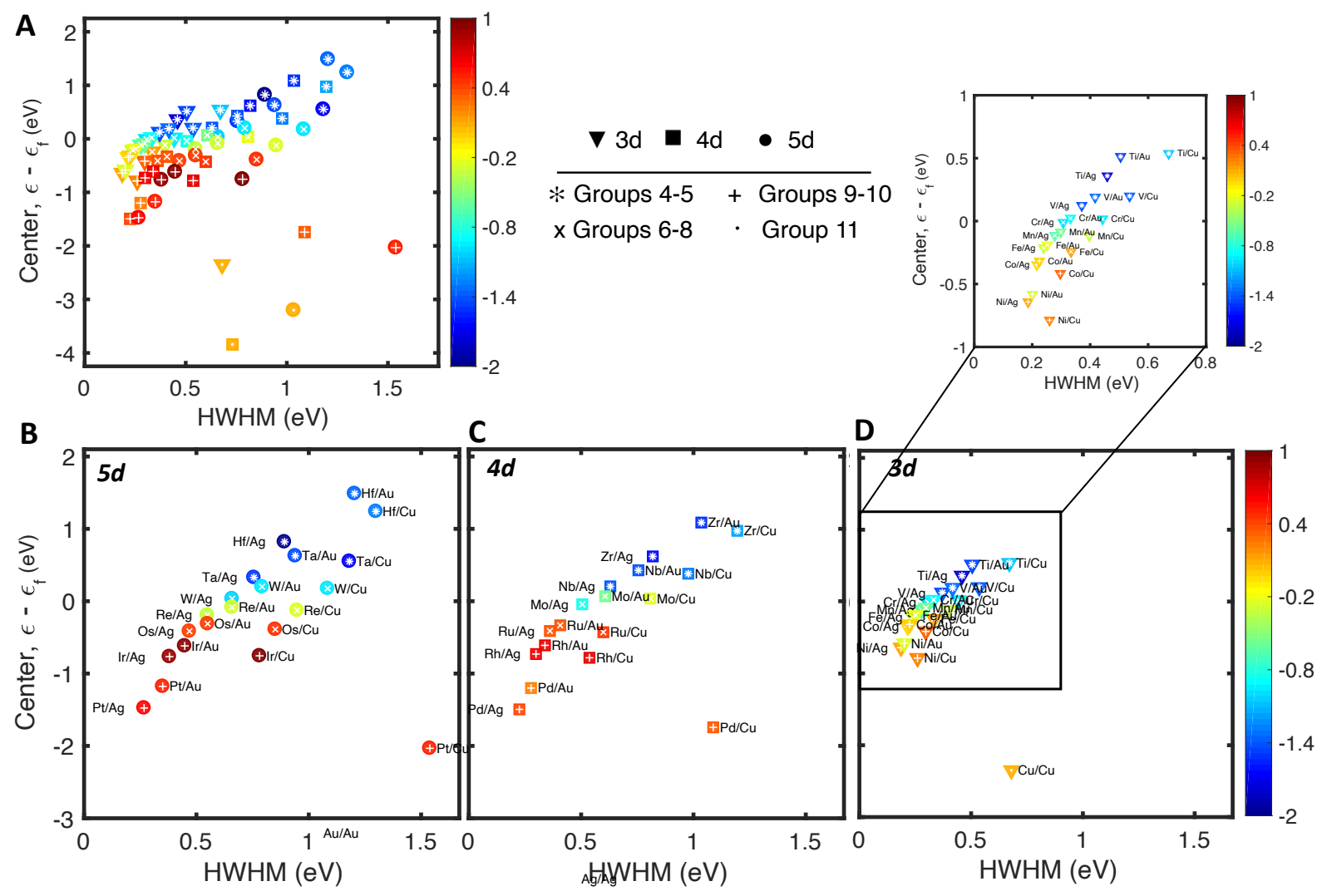

Figure S6: Visualization of selectivity maps using DFT-calculated values for marker shading by $\Delta \Delta \mathrm{E}$ (color scale in $\mathrm{eV}$, identical to Figure S5). An overlay of all SAA systems is shown in (A), with period-specific selectivity maps for $5 d, 4 d$, and $3 d$ SAAs shown in (B)-(D) respectively for comparison with Figure S5. Inset: Enlarged region containing most $3 d$ SAAs. Legend applies to all panels. A parity plot between the model and all data points above is given in Figure $4 \mathrm{~B}$ of the main text. 

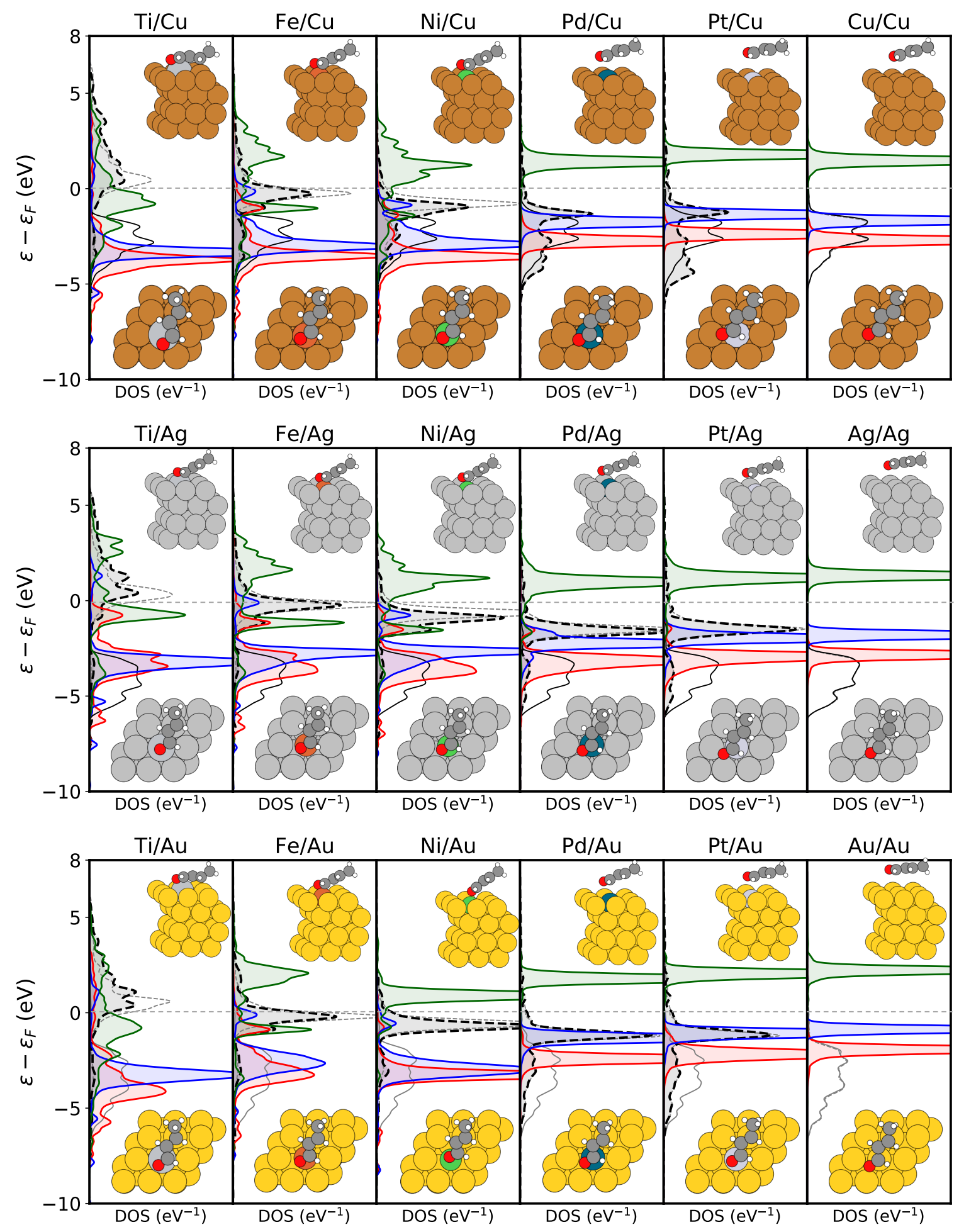

Figure S7: Orbital pDOS for crotonaldehyde adsorption in ald-mode on representative subset of SAAs. MOs shown are the HOMO-1 (red), HOMO (blue), and LUMO (green). The guest atom $d$ pDOS is shaded gray (dotted lines), while host is unshaded. Faint gray curves represent the adsorption site atom prior to adsorbate interaction. Insets: Stable adsorption geometry views, side and top. 

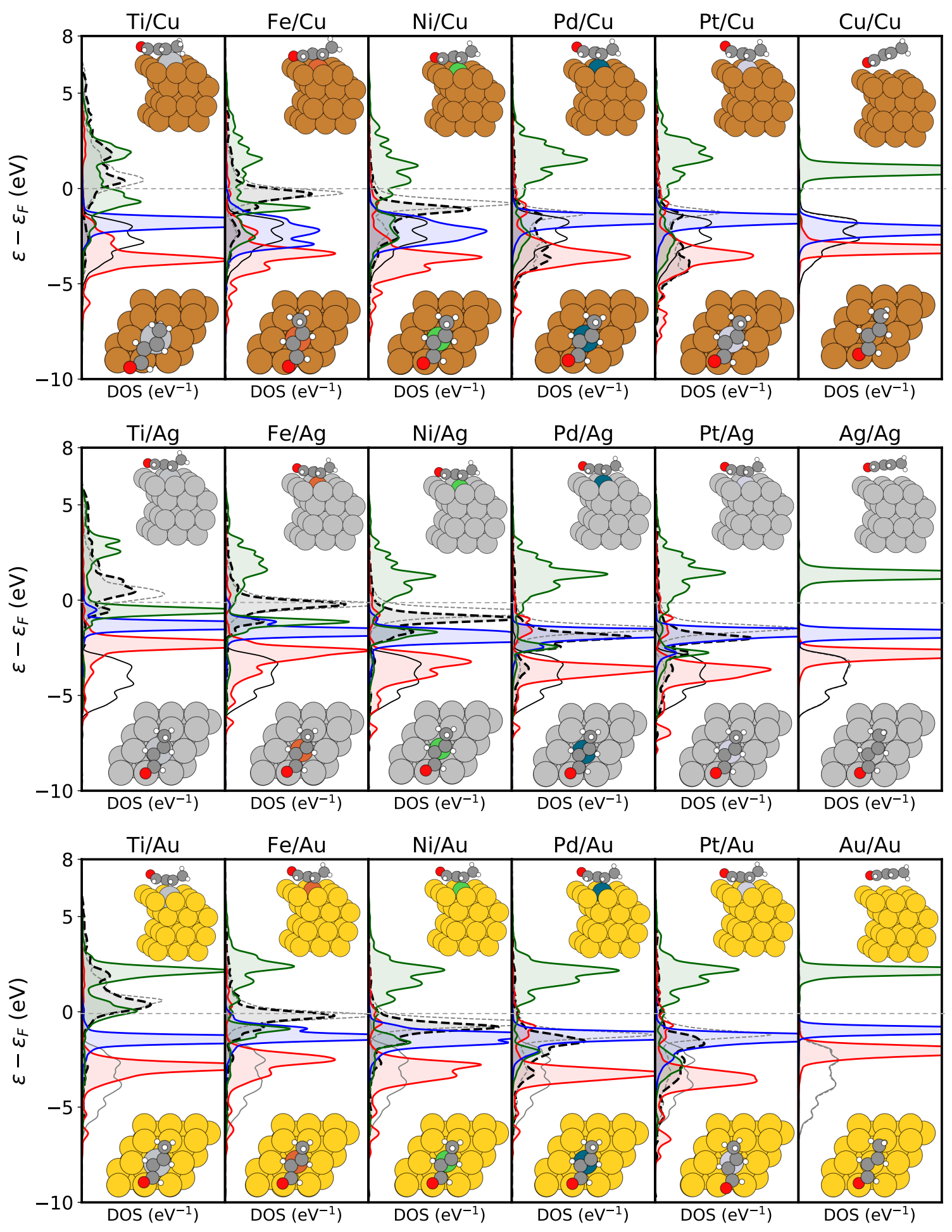

Figure S8: Orbital pDOS for crotonaldehyde adsorption in ene-mode on representative subset of SAAs. MOs shown are the HOMO-1 (red), HOMO (blue), and LUMO (green). The guest atom $d$ pDOS is shaded gray (dotted lines), while host is unshaded. Faint gray curves represent the adsorption site atom prior to adsorbate interaction. Insets: Stable adsorption geometry views, side and top. 
A

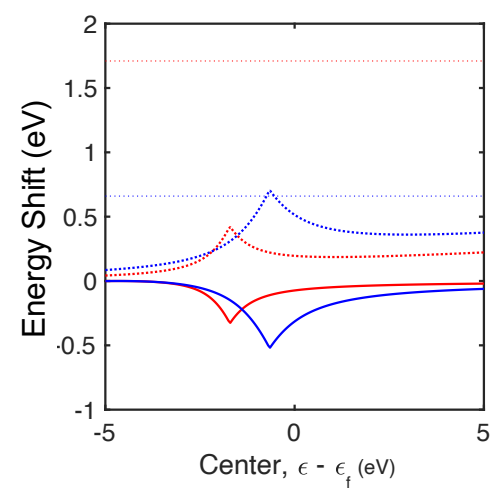

B

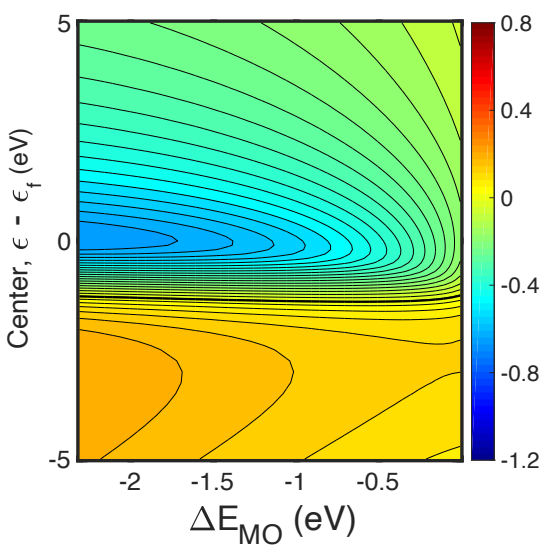

C

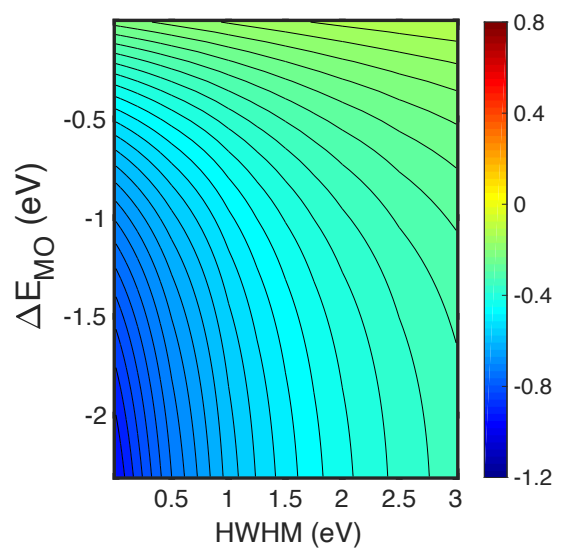

Figure S9: Illustrations of general principles and extensibility to other systems with differing separations in the molecular orbital energies. (A) Energy shift values representing the net upward or downward shift relative to the initial upper and lower energy states in a representative pure twolevel model while varying $d$-band center. Resonance peaks in the plot of energy shift vs. $d$-band center position illustrate the enhanced interactions of energetically aligned states. The initial orbital energies and coupling element values are chosen for the crotonaldehyde molecule $\left(V_{\text {HOMO }}^{\text {ald }}>\right.$ $V_{H O M O-1}^{\text {ald }}$ ), with $S=-0.2 \mathrm{~V}$, but the qualitative features of the plot exist for any choice of parameters. This is explored in the subsequent panels for differing MO separations separation, $\Delta \mathrm{E}_{\mathrm{MO}}$. (B) Difference in $d$-band interaction $\left(\Delta E_{\text {Номо }}-\Delta E_{\text {НОмО-1 }}\right)$ vs. $d$-band center and initial HOMO-1 energy using NAG model at constant Lorentzian HWHM $(0.75 \mathrm{eV})$ while holding HOMO energy and all coupling elements constant to physically realistic values (crotonaldehyde parameters). (C) Same measure of orbital discrimination varying the width at constant $d$-band center $(0 \mathrm{eV})$.

A

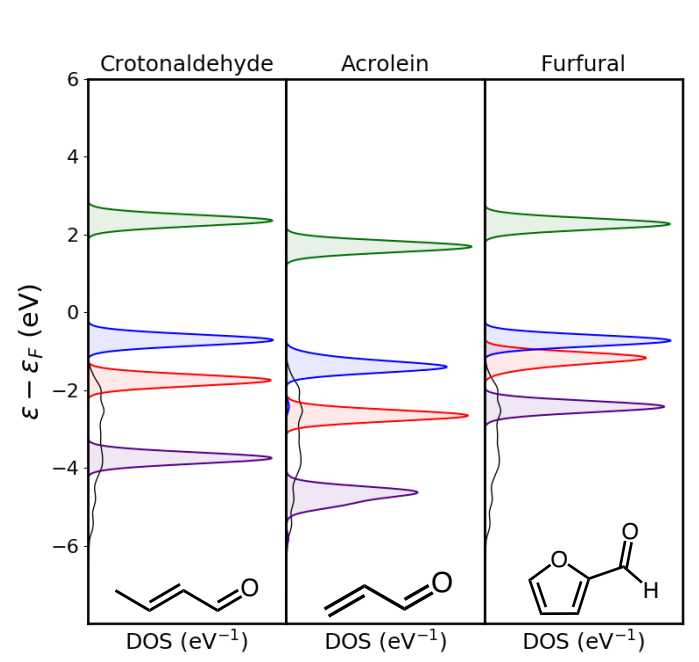

B

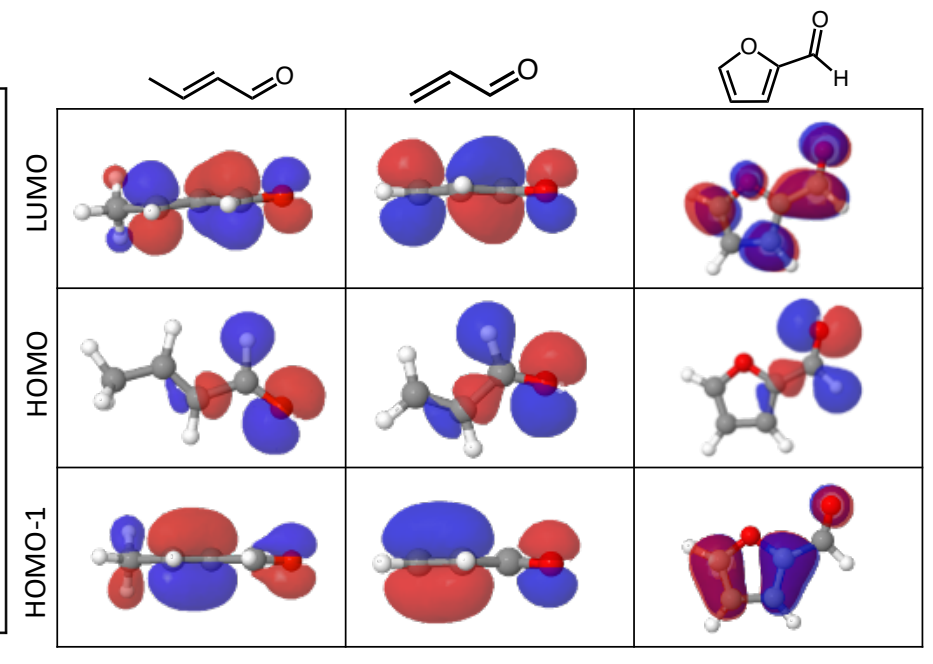

Figure S10: Comparison of frontier and adjacent orbitals showing generality of selectivity principle for several $\alpha, \beta$-unsaturated aldehydes. (A) Projected DOS for HOMO-2 through LUMO for crotonaldehyde, acrolein, and furfural, each computed at separation of $5 \AA$ from a $A u(111)$ surface. (B) Electron density contours showing the HOMO-1 and HOMO are always localized on alkene and aldehyde. 
A

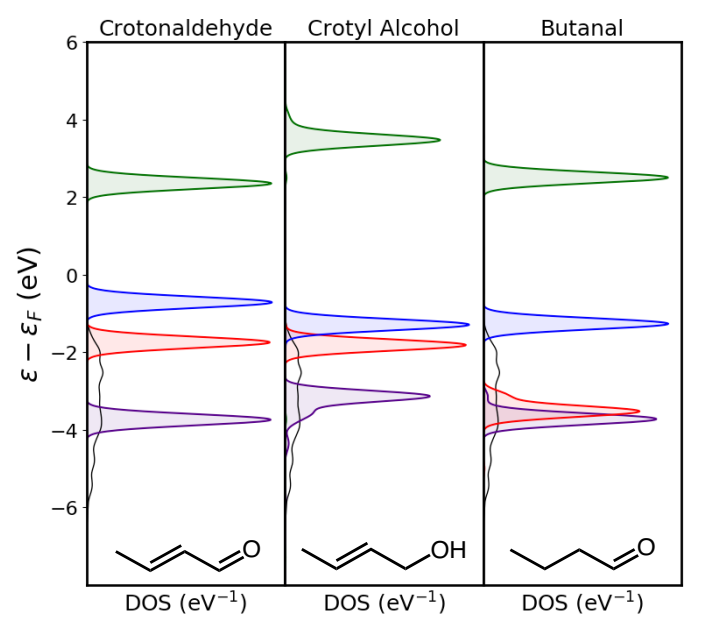

B

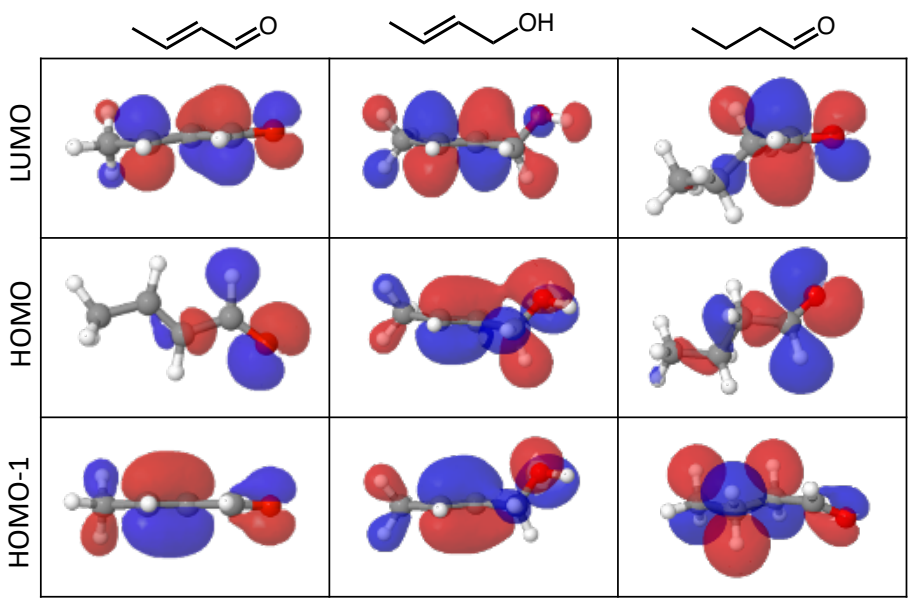

Figure S11: Comparison of frontier and adjacent orbitals for key intermediates in crotonaldehyde reduction. (A) Projected DOS for HOMO-2 through LUMO for crotonaldehyde, crotyl alcohol, and butanal, each computed at separation of $5 \AA$ from a $\mathrm{Au}(111)$ surface. (B) Electron density contours showing the respective spatial distributions of the orbitals. Practically, it could be concluded that the HOMO's of both crotyl alcohol and butanal, centered on the alkene and aldehyde respectively, will present challenges in maintaining orbital selectivity at high conversion, since these lie closer to the MOs of crotonaldehyde whose reduction yield these respective products and thus they would compete for adsorption more effectively than the competing orbital of crotonaldehyde itself. Nonetheless, band shape can bias adsorption toward crotonaldehyde. 
A

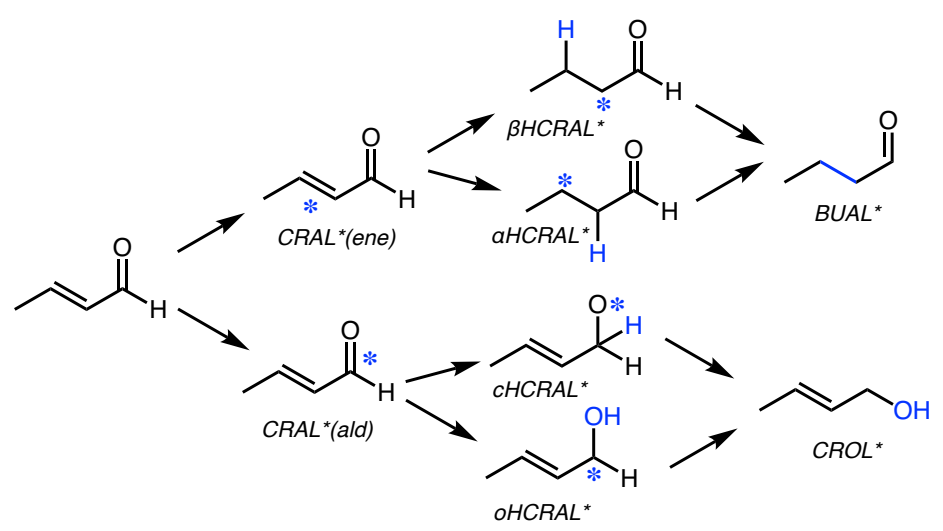

B

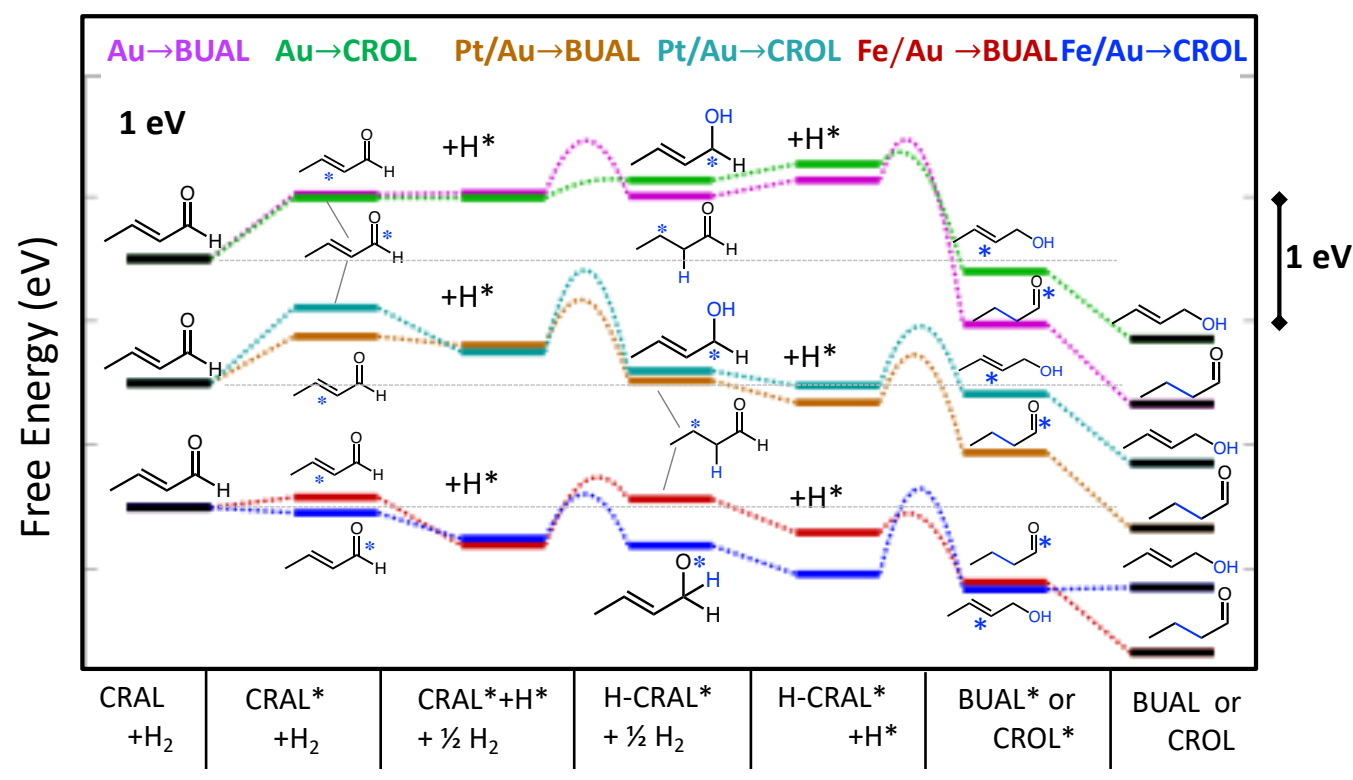

Figure S12: (A) Reaction network with different elementary hydrogenation paths considered. (B) Standard free energies for most favorable paths to crotyl alcohol and butanal on $\mathrm{Au}, \mathrm{Fe} / \mathrm{Au}$, and $\mathrm{Pt} / \mathrm{Au}$ (111) SAAs at $298 \mathrm{~K}$. The monometallic Au surface shows a strong preference for the crotyl alcohol path, but, consistent with prior experiment, weak substrate adsorption and $\mathrm{H}$-adsorption prevent the pathway from producing crotyl alcohol at high rates. Fe/Au permits a barrierless introduction of surface $\mathrm{H}^{*}$ while also allowing selective hydrogenation on the pathway to crotyl alcohol, albeit with a smaller difference in barrier over the butanal path than is seen for pure Au. $\mathrm{Pt} / \mathrm{Au}$ show more facile activation of the alkene group toward formation of butanal. 


\section{Method details}

\section{DFT calculations}

DFT calculations were performed with the projector augmented wave (PAW) approach ${ }^{1}$ using the GPAW software package (v. 1.5.2). ${ }^{2,3}$ Exchange and correlation energies were described by the Perdew-Burke-Ernzerhof (PBE) functional ${ }^{4}$, and an energy cutoff of $500 \mathrm{eV}$ was used for the plane wave basis set. Spin polarization was not included. All surfaces were modeled as $3 \times 3 \times 4$ fcc(111) periodic supercells separated by $20 \AA$ of vacuum. Dipole interactions between periodic slabs were removed with a dipole layer in the vacuum region. Geometries were optimized by fixing the two lowest subsurface layers at their optimized bulk lattice positions, while the two top layers and adsorbate atoms were relaxed until forces on all atoms were below $0.05 \mathrm{eV} / \AA$. Brillouin zone sampling was performed on a (6x6x1) k-point, gamma-shifted Monkhorst-Pack grid. ${ }^{5}$ The number of bands in each calculation was selected to be $25 \%$ larger than the number of occupied states. Projected densities of states are presented by summing the square moduli of the overlaps between the Kohn-Sham eigenstates and designated orbitals, multiplied by a Gaussian smearing of $0.2 \mathrm{eV}$. Transition state structure searches used the climbing image nudged elastic band (CI-NEB) method. ${ }^{6}$ Transition state atomic forces were converged to the same $0.05 \mathrm{eV} / \AA$ force criterion and were confirmed to have a single imaginary vibrational mode along the reaction coordinate.

Adsorption energies were determined by considering multiple initial adsorption geometries, performing structure optimization, and identifying the most stable species. The electronic contribution to energy changes was calculated for each system as:

$$
\Delta E_{D F T}=E_{\text {system }}-E_{\text {slab }}-E_{\text {adsorbates }}
$$

Free energy changes between various surface states were then computed according to:

$$
\Delta G=\Delta E_{D F T}+\Delta E_{Z P V E}+\Delta E_{v i b}-T \Delta S_{v i b}
$$

where EZPVE $_{\text {is }}$ the zero-point vibrational energy, $\mathrm{E}_{\text {vib }}$ is the internal energy of vibration at $298 \mathrm{~K}$ (within a harmonic normal mode analysis), and $\mathrm{TS}_{\mathrm{vib}}$ is the vibrational entropy of the adsorbed species at $298 \mathrm{~K}$, calculated with standard thermodynamic expressions. ${ }^{7}$ Free energies of gas phase species are calculated by including PV, translational, rotational, and vibrational energy and entropy terms, treating each species as an ideal gas at $1 \mathrm{~atm}$.

\section{Newns-Anderson-Grimley Model}

The NAG model was used to describe molecular adsorption via coupling interactions between an adsorbate orbital and a spectrum of surface states. We compare interactions between a surface and two different LCAO orbitals. Specific geometric considerations and bands structures that parameterize the model for the case of crotonaldehyde adsorption on SAA sites are discussed further below after highlighting the general features.

\section{(i) Background:}

Full derivation of the NAG adsorption model can be found in the original works and reviews. ${ }^{8-10}$ Within this framework, adsorption energies can be determined using the change in one electron energy levels based on the induced density of states $(\delta \rho)$ upon adsorption. While this is not appropriate to calculate absolute binding energies, it has been illustrated that the change in one electron energies is sufficient for capturing differences in binding energies across similar systems. ${ }^{11}$ Neglecting spin for simplicity, the adsorption energy for each system (for which only differences between systems is compared) is given by: 


$$
\Delta E_{a d s}=\int_{-\infty}^{\varepsilon_{f}} \delta \rho \cdot \varepsilon d \varepsilon-\Delta N \varepsilon_{f}+n_{a}\left(\varepsilon_{f}-\varepsilon_{a}\right)
$$

where $\Delta N=\int_{-\infty}^{\varepsilon_{f}} \delta \rho d \varepsilon$ indicates the change in number of filled states due to introduction of the adsorbate (the hybridized states are counted in the induced surface DOS but are not in the initial state surface DOS), and $n_{a}$ is the number of electrons contributed by the adsorbate state originating at energy $\varepsilon_{a}$. In contrast to a two-level model, surplus or deficit charge is always donated to or taken from the Fermi level $\left(\varepsilon_{f}\right)$, and so the terms involving $\varepsilon_{f}$ represent moving the $n_{a}$ electrons from the adsorbate state to the Fermi level, then redistributing them from the Fermi level into the induced density of states. An additional on-site Coulomb term is often included to capture the effect of changes to the energy of the adsorbate level from changes to its occupancy as it approaches the surface, but in this work, we extract $\varepsilon_{a}$ directly from DFT calculations (taking orbital level several $\AA$ outside the surface where it only interacts with the $s p$-electron density) such that the alignment is implicit.

To compute the induced DOS, a chemisorption function $(\Delta)$ and its Hilbert transform $(\Lambda)$ are defined as:

$$
\begin{gathered}
\Delta\left(\varepsilon, \varepsilon^{\prime}\right)=\pi \sum_{k}\left|\varepsilon \cdot S_{a k}-V_{a k}\right|^{2} \delta\left(\varepsilon^{\prime}-\varepsilon_{k}\right) \\
\Lambda(\varepsilon)=\mathcal{P} \frac{1}{\pi} \int_{-\infty}^{\infty} \frac{\Delta\left(\varepsilon, \varepsilon^{\prime}\right)}{\varepsilon-\varepsilon^{\prime}} d \varepsilon^{\prime}
\end{gathered}
$$

where $S_{a k}$ and $V_{a k}$ are the overlap and coupling matrix elements between the adsorbate and each surface state, and $\mathcal{P}$ indicates the Cauchy principal value. Eqns. (4) and (5) define the complex self-energy $q(\varepsilon)=\Lambda(\varepsilon)-i \Delta(\varepsilon)$, where $\Delta(\varepsilon) \equiv \Delta(\varepsilon, \varepsilon)$. Integration of the induced density of states can then be cast in terms of $\Delta$ and $\Lambda$ with a phase shift function $\eta(\varepsilon)$, defined such that $\delta \rho(\varepsilon)=-\frac{1}{\pi} \frac{d \eta(\varepsilon)}{d \varepsilon}$. This leads to:

$$
\Delta E_{a d s}=\frac{2}{\pi} \int_{-\infty}^{\varepsilon_{f}} \eta(\varepsilon)-n_{a}\left(\varepsilon_{a}-\varepsilon_{f}\right)=\frac{2}{\pi} \int_{-\infty}^{\varepsilon_{f}} \tan ^{-1}\left[\frac{\Delta(\varepsilon)}{\varepsilon-\varepsilon_{a}-\Lambda(\varepsilon)}\right]-n_{a}\left(\varepsilon_{a}-\varepsilon_{f}\right)
$$

where the factor of 2 is present for spin degeneracy, and the phase shift ( $\tan ^{-1}$ term) is taken on the interval $-\pi$ to 0 whenever localized states do not exist outside the surface DOS $^{8}$ - this will always be the case when broad $s p$-state contributions are also included, ${ }^{12}$ as we do in this work.

The adsorbate-localized DOS can also be found according to:

$$
\rho_{a}(\varepsilon)=\frac{1}{\pi} \frac{\Delta(\varepsilon)}{\left[\varepsilon-\varepsilon_{a}-\Lambda(\varepsilon)\right]^{2}+\Delta(\varepsilon)^{2}}
$$

Equation (7) can be interpreted straightforwardly in the limits of narrow or wide bands. The $\Lambda(\varepsilon)$ term, defined in Eq. (5), represents an energy shift, which in the limit of strong coupling to narrow bands (e.g. $d$-states) produces bonding and antibonding levels. Inspection of Eq. (7) reveals that bonding and antibonding resonances will appear at the intersections of $\varepsilon-\varepsilon_{a}$ with $\Lambda$. For nearly flat bands (sp-metals), $\Lambda \rightarrow 0$ and the broadening approaches a simple Lorentzian form with fixed HWHM given by $\Delta$. Coupling matrix elements defining the chemisorption function and other relationships above are next discussed.

(ii) Matrix elements

It was shown by Newns ${ }^{8}$ that the average coupling element with each band can be expressed in terms of the coupling elements with localized $d$-orbitals according to: $\left|V_{a k}\right|^{2}=$ $\sum_{d_{i}}\left|V_{a d_{i}}\right|^{2}$. The summation gives an overall $d$-state coupling element, which can be taken from solid state tables ${ }^{13}$ : 


$$
V_{l d m}=\eta_{l d m} \frac{\hbar^{2}}{m} \frac{r_{d}^{3 / 2}}{d^{7 / 2}}
$$

where $r_{d}$ is the tabulated extent of the $d$-orbital, $d$ is the separation with the adsorbate orbitral, $\frac{\hbar^{2}}{m}=$ $7.62 \mathrm{eV} \cdot \AA^{2}$, and $\eta$ is an orbital shape factor determined by the symmetry and bond type: $\eta_{s d \sigma}=$ $-3.16, \eta_{p d \sigma}=-2.95$, and $\eta_{p d \pi}=1.36$. When the angular orientations of the interacting orbitals deviate from the internuclear axis, the coupling is cast as a linear combination of $\sigma$ and $\pi$ interactions using relations between the appropriate spherical hamonics as tabulated by Slater and Koster. ${ }^{14}$ These angular dependences are illustrated in Figure S13. The coupling elements are further approximated as being independent of energy, as is common in the NAG modeling approach. The chemisorption function for the $d$-band interaction is thus given by:

$$
\Delta_{d}\left(\varepsilon, \varepsilon^{\prime}\right)=\pi|\varepsilon \cdot S-V|^{2} \rho_{M}\left(\varepsilon^{\prime}\right)
$$

with $\rho_{M}$ being the metal site $d$-DOS. The overlap matrix elements are approximated as proportional to the coupling elements: $S=-\alpha V$, where the term $\alpha$ is commonly treated as a fitting parameter. ${ }^{15-17}$ We use a typical value $\alpha=0.2$.

The surface $d$-state DOS was modeled as a Lorenztian function defined by the $d$-band center and the HWHM of the $d$-band (the second moment is undefined for a Lorentzian and thus not representative of width). This functional form was found to most accurately fit the pDOS for isolated atoms in SAAs, which may be expected based on their weak interactions with the host (c.f. Eq. 7). Other distributions including Gaussian and semi-elliptic were also considered and give the same qualitative effects with respect to the influence of width on bonding mode preference, all other factors being equal. This analysis is shown in Figure S4. When attempting to relate the model to specific atomic elements (as in Figure S5C-E and Figure 4B of the main text), the coupling matrix elements were further scaled by the ratio between the element of interest and a reference case (decribed below); this relates to the differences in $d$-radii between different elements per Eq. 8: $\frac{V_{A}^{2}}{V_{B}^{2}}=\frac{r_{d A}^{3}}{r_{d B}^{3}}$. The scaling values are shown in Figure S5A and were assigned to each Lorentzian $d$-band by interpolation according to the filling (integral up to Fermi level) using elements of similar filling in a given period $(3 d, 4 d, 5 d)$. Finally, $s p$-interactions are included through a constant, $\Delta_{0}$, since these states are reasonably described by a flat band approximation, leading to an overall chemisorption function $\Delta=\Delta_{d}+\Delta_{0} \cdot{ }^{12,18}$ This addition does not directly influence the impact of varying the $d$-band, but allows use of Eq. (6) without creating special cases for localized states.

\section{(iii) Application to SAA selectivity problem}

To understand the propensity of various SAAs to couple with specific MOs, adsorbate geometries for each binding mode were selected for a reference material, with changes to the surface electronic structure viewed as a perturbation relative to this case. $\mathrm{Co} / \mathrm{Au}$ was selected as the reference state because this SAA shows nearly identical binding energies in each adsorption mode; perturbing about the point of equal affinities was viewed as the best way to identify the factors that govern selective preferences without too large of extrapolations in the simple model. While perturbation of the electronic structure should cause adjustment to the adsorbate geometry in response (e.g. changing bond distance), it may be noted that the largest contribution to adsorbate bond energy on transition metals comes from an attractive $s p$-interaction that is roughly constant across these systems. The $s p$-interaction is the predominant determiner of bond distances as the adsorbate seeks a region of optimal electron density. ${ }^{19}$ The $d$-state contribution can then be 
considered a small perturbation which modulates the bonding, though it still governs trends in adsorption energy across the transition metals. Thus, we acknowledge using the geometry on a $\mathrm{Co} / \mathrm{Au}$ surface as the reference geometry will be most representative of Au-hosted, late $3 d$-metal SAA systems (optimal $s p$-density at similar geometries, similar $d$-orbital radii), but the trends (as in Figure 4 of the main text) and governing physical phenomena should still be well-capturedi.e. stabilization or destabilization of one binding mode relative to another in response to a perturbation is the primary output being sought. Differences in binding energy relative to those on the reference material should not be viewed as absolute, but their qualitative ordering is expected to hold after any adjustment in geometry that would occur.
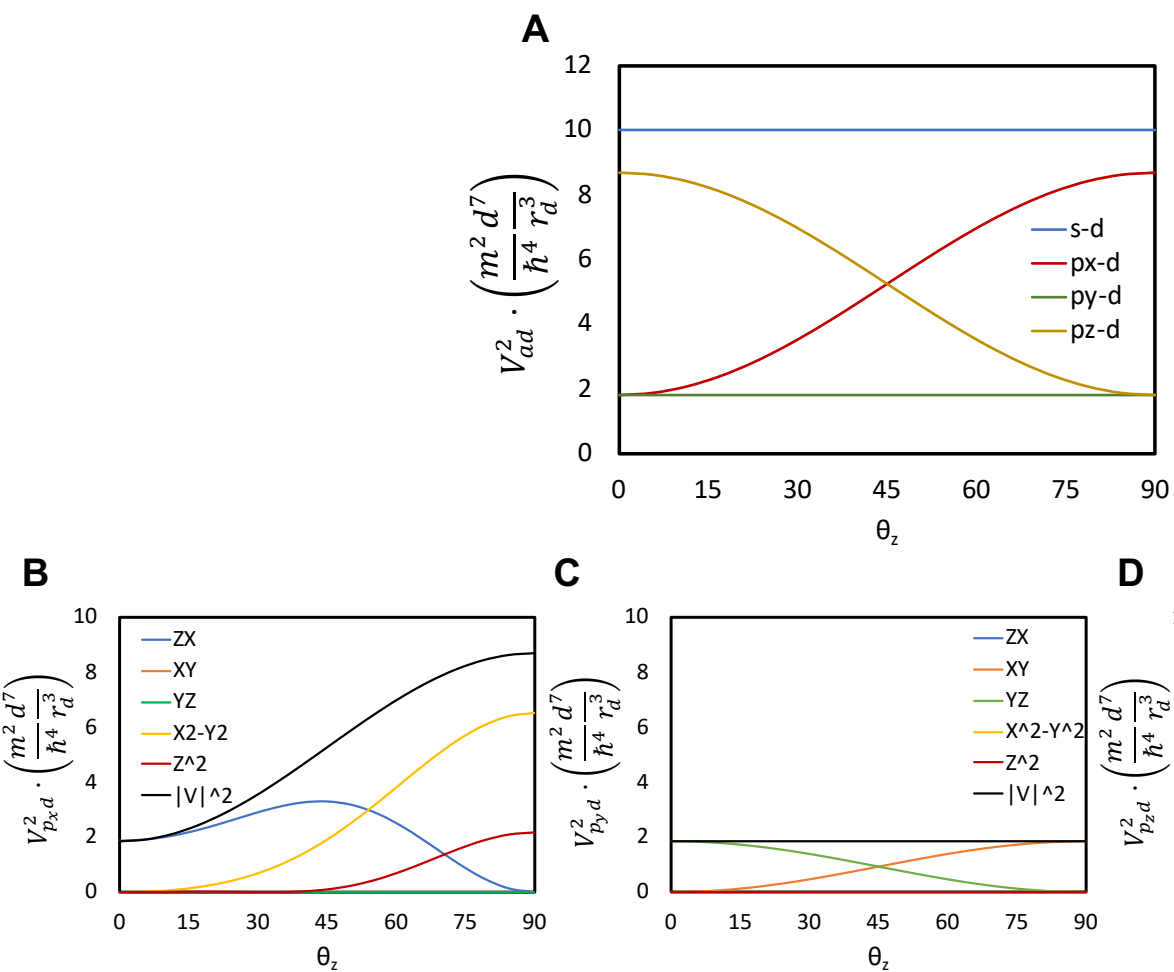

C
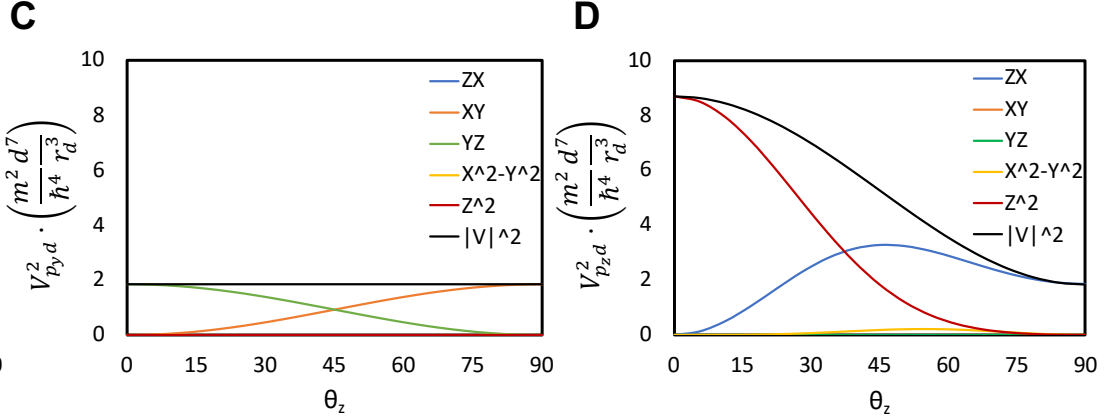

Figure S13: Coupling matrix elements computed according to tight-binding framework solid state tables ${ }^{13}$ using orientational factors tabulated by Slater and Koster. ${ }^{14}$ (A) Total coupling element with $d$-states $V_{a d}$ for atomic $s$ and $p$ orbitals at fixed distance and orientation, rotating through $90^{\circ}$ about the $y$-axis. (B) Coupling element for $p_{z}$ state with each $d$-orbital and total coupling, rotating through $90^{\circ}$ about the $y$-axis.

To determine the coupling elements with LCAO molecular orbitals of crotonaldehyde, the MOs were first computed in a minimal atomic basis set so they could be approximately decomposed in terms of atom-centered $s$ - and $p$-orbitals. These were shown schematically in Figure S2 with the squared LCAO coefficients tabulated. Defining the $x y$-plane as that of the $s p^{2}$ system of crotonaldehyde, the HOMO-1 is predominantly composed of a bonding combination of two $p_{z}$-orbitals as well as some density on the $\mathrm{O} p_{z}$-orbital, while the HOMO is predominantly on the $p_{y}$ nonbonding orbital of the carbonyl group oxygen, with a small contribution from $p_{x}$ and minor contributions from the aldehyde $\mathrm{H}$ and an $s p^{2}$-orbital on the alpha carbon. The LUMO mainly contains $p_{z}$ orbitals from oxygen and the three adjacent carbons. The approximate wavefunctions (only terms with squared coefficient $>0.04$ ) are shown below: 


$$
\begin{gathered}
\psi_{\text {номо-1 }} \approx\left\{0.542 p_{z}^{(C 3)}+0.534 p_{z}^{(C 2)}\right\}-\left[0.462 p_{z}^{(O)}\right] \\
\psi_{\text {номо }} \approx\left[0.796 p_{y}^{(O)}-0.471 p_{x}^{(O)}\right]-0.358 s^{(H 1)}-0.219 s^{(C 2)}+0.269 p_{y}^{(C 2)} \\
\psi_{\text {LUMO }} \approx\left\{-0.652 p_{z}^{(C 3)}+0.322 p_{z}^{(C 2)}\right\}+\left[0.570 p_{z}^{(C 1)}-0.623 p_{z}^{(O)}\right]
\end{gathered}
$$

Each atomic orbital (scaled by appropriate LCAO coefficients) was considered to interact with $d$ orbitals in the respective binding modes, with overlap between LCAO contributions remote from the reaction site assumed to be neglegible. Thus terms grouped by " \{\} " braces above were considered in the ene-mode of binding, while terms with "[]" brackets were considered in the aldmode binding.

Recalling that the goal is to capture interaction with a metal site of arbitrary characteristics (with all other factors being equal), we computed coupling elements for the reference geometry of crotonaldehyde bonding to the Co site of an Co/Au SAA in either ald-mode or ene-mode. The relevant orbital interactions are highlighted in Figure S14, and coupling elements were computed from Eq. (8), where an $r_{d}$ value of $0.76 \AA$ represents $\mathrm{Co}$, and the angular coordinates were accounted by use of the tabulated relations by Slater and Koster. ${ }^{14}$ We note that the orientations of the adsorbate orbitals have only a small effect on the value of the coupling matrix elements. This is due to partial compensation effects while transitioning between $\sigma$ and $\pi$ contributions with the various $d$-orbitals as the bond angle is changed as well as the similar magnitudes of the associated shape factors. Referring back to Figure S13, we see (i) values of the various coupling elements $\left(V_{s d}, V_{p x d}, V_{p y d}\right.$, and $\left.V_{p z d}\right)$ while moving each orbital through an $\operatorname{arc}$ of $90^{\circ}$ at fixed orientation relative to the surface atom and (ii) a decomposition of the coupling elements between each $p$ orbital and the five $d$-orbitals. (Note that the plots in Fig. S13 are only to illustrate the interaction of constituent atomic orbitals and do not directly represent the LCAO states-for example, while each $p_{z}$ orbital of the HOMO-1 overlaps the $d_{x z}$ surface state, their in-phase combination has net zero overlap). Collectively this illustrates that small perturbations in the adsorbate geometry will not qualitatively alter the relative interaction strengths with the MOs. Using the coupling elements determined in the aformentioned manner, the NAG model is fully defined and used to generate Figures 3, 4, S3-5, and S9, with modifications noted where applicable.
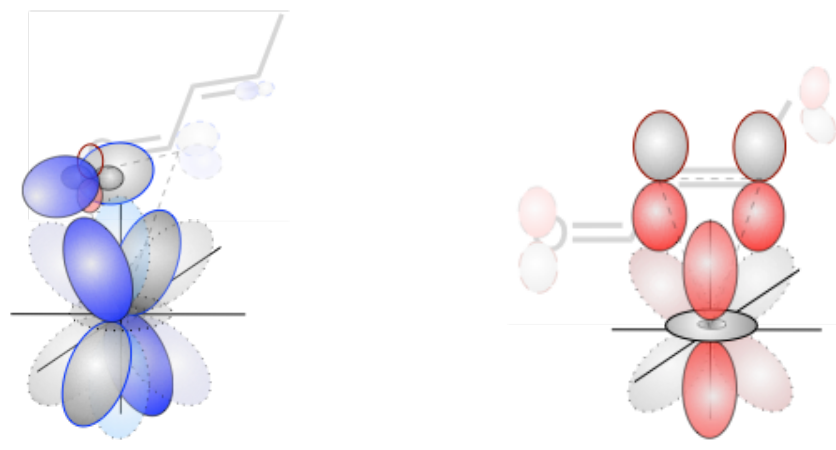

Figure S14: LCAO MOs of crotonaldehyde shown as full schematics above and with the relevant component interactions with $d$-orbitals below, as discussed in the methods. 


\section{References}

1. Blochl, P. E. Projector augmented-wave method. Phys. Rev. B 50, (1994).

2. Enkovaara, J. et al. Electronic structure calculations with GPAW: A real-space implementation of the projector augmented-wave method. J. Phys. Condens. Matter 22, (2010).

3. Mortensen, J. J., Hansen, L. B. \& Jacobsen, K. W. Real-space grid implementation of the projector augmented wave method. Phys. Rev. B - Condens. Matter Mater. Phys. 71, 1-11 (2005).

4. Perdew, J. P., Chevary, J. A., Vosko, S. H., Jackson, K. A., Pederson, M. R., Singh, D. J. \& Fiolhais, C. Atoms, molecules, solids, and surfaces: Applications of the generalized gradient approximation for exchange and correlation. Phys. Rev. B 46, 6671-6687 (1992).

5. Monkhorst, H. J. \& Pack, J. D. Special points for Brillouin-zon integrations. Phys. Rev. B 13, 5188-5192 (1976).

6. Henkelman, G., Uberuaga, B. P. \& Jónsson, H. Climbing image nudged elastic band method for finding saddle points and minimum energy paths. J. Chem. Phys. 113, 9901-9904 (2000).

7. Atkins, P. \& Paula, J. de. Physical Chemistry: Thermodynamics, Structure, and Change. (Oxford University Press, 2014).

8. Newns, D. M. Self-consistent model of hydrogen chemisorption. Phys. Rev. 178, 11231135 (1969).

9. Muscat, J. P. \& Newns, D. M. Chemisorption on metals. Prog. Surf. Sci. 9, 1-43 (1978).

10. Grimley, T. B. Chemisorption Theory. Prog. Surf. Membr. Sci. 9, 71-161 (1975).

11. Nørskov, J. K. Chemisorption on metal surfaces. Reports Prog. Phys. 53, 1253-1295 (1990).

12. Wang, S., Pillai, H. S. \& Xin, H. Bayesian learning of chemisorption for bridging the complexity of electronic descriptors. Nat. Commun. 11, 1-7 (2020).

13. Harrison, W. A. Electronic Structure and the Properties of Solids: The Physics of the Chemical Bond. (Dover Publications, 1989).

14. Slater, J. C. \& Koster, G. F. Simplified LCAO method for the periodic potential problem. Phys. Rev. 94, 1498-1524 (1954).

15. Hammer, B., Norskov, J. K., Science, E. S., Physics, A. M. \& Technology, A. Electronic factors determining the reactivity of metal surfaces. Surf. Sci. 343, 211-220 (1995).

16. Xin, H. \& Linic, S. Communications: Exceptions to the d-band model of chemisorption on metal surfaces: The dominant role of repulsion between adsorbate states and metal d-states. J. Chem. Phys. 132, 221101 (2010).

17. Xin, H., Holewinski, A. \& Linic, S. Predictive Structure-Reactivity Models for Rapid Screening of Pt-Based Multimetallic Electrocatalysts for the Oxygen Reduction Reaction. ACS Catal. 2, 12-16 (2012).

18. Nørskov, J. K. Effective medium potentials for molecule-surface interactions: $\mathrm{H} 2$ on $\mathrm{Cu}$ and Ni surfaces. J. Chem. Phys. 90, 7461-7471 (1989).

19. Hammer, B. \& Norskov, J. K. Theoretical surface science and catalysis--calculations and concepts. Adv. Catal. 45, 71-129 (2000). 